last few days seen, with Dr. Reynolds, a case of rery highlymarked diastolic basic murmur, in which, although the patient cannot be said to be positively anæmic, a well-defined thrill attends the reflux through the aortic orifice. This diastolic thrill is better conducted upwards, above the heart's base, than, as the connected murmur, downwards towards the ensiform cartilage. It is not unworthy of note, that though the murmur is extremely loud at the base, a perfectly well-defined second sound, without murmur, is heard at the left apex." "

Now, there are in this singular case two points of interest: one that I can understand, and one that I must say I cannot. I can understand why convection should bring the basic murmur down towards the ensiform cartilage, and leave the thrill, with which it was connected, at the base, conducted to the chest-wall in the immediate neighbourhood of its production. But I cannot understand how, with a regurgitant murmur, and the blood flowing down from the aorta into the ventricle towards the apex, a well-defined second sound should be heard there without murmur.

It is in this, to me, unintelligible part that this case resembles that of Jessie $\mathrm{H}-$. It is just the reverse of what I should have thought the laws of convection would have brought about, and just the reverse of what they did bring about in the case of $W-$, which $I$ have already related to you, in the case of my private patient, and in the case of Harriet $\mathrm{S}-$. In the case of $\mathrm{W}-$, if you remember, which was one of mitral disease, a natural first sound was heard at the apex unattended by murmur, while a reguroitant murmur, unattended by the natural element of the first sound, was heard in the axilla and back-that is, the natural valve-sound was heard at the part from which the blood was flowing, while the murmur was heard near the cavity to which it was flowing. In this case of Dr. Walshe's, and in that of Jessie $\mathrm{H}-$, the murmur was heard at the part from which the blood was flowing (the aortic orifice), and the natural sound at that to which it was flowing (the ventricle).

If the murmur had been heard at the base together with the second sound, and the murmur alone at the apex with. out any second-sound element, I could have understood it; because I could have understood that the refluent blood might have carried the murmur to the apex by convection, though conduction might have failed to transmit so far the shock of the closing aortic valves.

The only explanation that I can imagine of it is, that the regurgitating stream was so slender that it did not reach the apex-that it was a mere fine squirting, through a narrow chink, of a score of drops or so, and that, perhaps, in a direction not towards the apex,- -and thus that convection was not engaged at all. The fact that the closure of the valves was sufficiently good to produce a natural second sound, audible at the apex, is almost a proof of this.

Is it possible that the regurgitation in these cases was through the pulmonic valves, and not the aortic? In Dr. Walshe's case, the fact that the murmur was audible at the lower sternum, and not at the left apex, would be compatible with such a supposition, great as are the probabilities against it in other respects. In the case of Jessie $\mathrm{H}-$ - I was unable, from the patient's suddenly wishing to leave the hospital, to make any further investigations.

What, gentlemen, I wish to impress upon you by this lecture, and by the cases to which I have directed your attention, is that the seat of a cardiac murmur is often displaced to a considerable distance from the seat of its generation, and that a frequent cause of this displacement is the agency of convection. Why a murmur should be so transferred in one instance and not in another, it is difficult to say. I find it at present impossible to say. 'The whole subject is involved in difficulty. The daily practice of critical auscultation reveals anomalies in cardiac murmurs that suggest interesting speculation at every step. Doubtless there are other factors concerned in these irregularities besides convection, involving, perhaps, anatomical considerations or peculiarities of morbid changes in individual cases. One of these days we may become as familiar with the law of these aberrant cases as we now are with that of what may be called normal murmurs. At some future time I hope to treat the whole subject of the Localisation of Cardiac Murmurs in a broader and more general way. In the meantime, you must accept these remarks as a contribution, as far as convection goes, towards such an object.

\section{ON THE USE OF CHLOROFORM IN THE CURE OF CLEFT PALATE.}

CASES ; WITH REMARKS.

Bх THOM A S SMITH, F.R.C.S.,

ASSISTANT-SURGEON TO ST. BARTHOLONEW'S, AND SURGEON TO THE HOSPYTAE FOR SICK CHYLDREY.

IN October, 1867, I presented to the Royal Medical and Chirurgical Society a paper on the "Cure of Cleft Palate by Operation in Children." In this I advocated the performance of the operation in early life, and described a species of gag by the aid of which the operation could be done under the influence of chloroform. A plan of operation was also described, more rapid of execution, and entailing less violence on the soft parts, than the method in ordinary use, and therefore more suited to the delicate tissues of young children. The advantages expected to result from the plan advocated were-1. The painless performance of the operation on patients of any age. 2. A much better result as regards articulation when the operation is done in early life than when the cure is postponed until the usual age for operation. 3. The more complete cure of clefts in the bony palate, owing to the more vigorous bone-producing property of the periosteum in early life. The data upon which these expectations were founded were derived from the results in eleven cases-all that I had at that time operated upon. The experience of myself and others during the last two years, and a much larger number of cases, have justified the expectations I formerly entertained.

Cleft soft palate.-The patient was a stunted childish girl, aged thirteen, who was sent to me by Dr. Priest, of Waltham $\Delta$ bbey. Her articulation was very imperfect; the entire soft palate was cleft. On March 27th, 1867, I operated under chloroform at St. Bartholomew's Hospital. Four fishing-gut sutures were introduced; the palato-pharyngeus and levator palati muscles were divided on both sides. The oirl was fed on fluid nourishment, and, as she seemed to flag, nutrient enemata were added. Eight days after the operation the sutures were withdrawn, the cleft being completely united and the symmetry of the palate perfect. Dr. Priest reports on Jan. 9th, 1868, the following:- " The girl $\mathrm{C}-$ continues to improve; her articulation is much better; she talks more distinctly; and $I$ am in hopes that in a few months, if she is careful, very little trace of her infirmity will remain."

Cleft soft palate.-S. B-, a girl, aged two years and eleven months, a healthy-looking child, was brought into the Children's Hospital on August 27th, 1867. There was a complete cleft of the soft palate; the hard palate was not involved. The child could scarcely speak. Food and fluids returned through the nostrils when the child swallowed very hastily. 'The edges were pared, and four sutures were passed, the two lower horsehair, the two upper very fine gut. The palato-glossus and palato-pharyngeus muscles were both notched. The levatores palati were cut; two oblique cuts were made above, as there was at this point some tension. The whole fitted exactly.

The next day (the second after the operation) the eruption of scarlatina appeared; soon afterwards the sutures separated, and the child left the hospital before convalescent, with the wound wholly disunited.

Oct. 11th.-I operated again, using two horsehair and one gut suture.

21st.-Mother reports that part of the palate is united.

Nov. 11th.-I saw the child, and the cure appeared to be complete, except a small hole, as big as a No. 6 shot,just in the middle of soft palate.

12th. - Two sutures withdrawn.

Jan. 9th, 1868.-A small hole about the size of a No. 2 catheter still remains in the centre of the soft palate. The mother reports that the child can "say anything." As this hole does not produce any sort of inconvenience, no further operative measure has been adopted.

L. A-_, aged three years and two months, a healthy 
country child, has a cleft of the entire soft palate, and of the posterior part of the hard.

June 1st, 1867. - The edges were pared, and three sutures were introduced, the two lower ones being of fishing gut, the upper of silver wire. Four days after the operation the child was seized with scarlatina; and a day or two later it was evident she had whooping-cough. For a few days the palate remained united; but subsequently it gave way in its whole extent.

Oct. 12th.-T again operated under chloroform; and four gut and one horsehair sutures were introduced. The palato-pharyngei were divided, and oblique cuts were made above.

Nov. 4th.-A considerable part of the velum has united. There still remains an opening in the roof of the mouth, measuring three quarters of an inch in its long diameter.

Dec. 16th. - I passed a horsehair suture through the uvula, and a gut suture in the upper part of the soft palate. Three days afterwards she was attacked with mumps. The uvula has united. The child's power of articulation has much improved.

June 6th, 1868.-The mother reports that she has "given up the idea of having anything more done to the palate, as the child gets on famously with her talking."

Double harelip; complete cleft of the hard and soft palate; eversion of the intermaxillary bone, and deficiency of the columna nasi.-Henry M-, aged five years, was admitted into the Children's Hospital August 20th, 1867. Four years before I had operated upon the harelip. At the date of the operation on his palate, the cleft in the alveolar process had united, and almost the anterior half of the divided hard palate had coalesced; there was therefore a cleft of the soft palate and of rather more than half of the hard.

The operation on the soft palate was performed under chloroform on August 20th. Five sutures were introduced, one of silver and four of fishing-gut. The levatores palati were divided, and small oblique incisions were made just at the upper part of the soft palate. Seven days afterwards the sutures were removed under chloroform. Three weeks afterwards the soft palate had united, except at its lowest part, and the uvula, which was still cleft.

Oct. $22 \mathrm{nd}$. - His articulation has greatiy improved; the hole between the hard and soft palate is diminishing.

June, 1869.-He has good power of articulation, except for sibilant sounds. There remains still a cleft in the bony palate, very narrow, but a full inch in length. He is to come to the hospital in August for the cure of this.

Cleft of soft and half the hard palate. - K. H-, a gir aged four years and eleven months, was recommended to me by Dr. West. The palate was widely cleft, ending in front in a rounded margin, and not running to an angle, as is usual. The palatine arch is high ; food and fluids occasionally return through the nostrils, unless great care is taken in deglutition. Considering the amount of deformity, the articulation is unusually good, though indistinct and guttural.

June 12th, 1869. - Under chloroform, the edges of the cleft were pared, the muco-periosteum was detached from the bony palate above, and eight sutures were passed, those in the soft palate being horsehair and the others fine silver wire.

The next day the child suffered severely from chloroform sickness. She only began to take nourishment effectively on the second day after the operation. On the sixth day four of the sutures were cut, and next day were removed. On the eighth day the remaining sutures were taken out.

23rd.- The patient returned to the country with a small round hole at the junction of the hard and soft palate, and a little chink at the anterior angle of the fissure.

July 3rd.-The hole in the soft palate has closed, and there remains a very small opening at the anterior angle of the cleft in the hard palate.

Cleft of hard and soft palate.-Annie W- aged eleven years, admitted into St. Bartholomew's Hospital on the 21st of September, 1868. She was a feeble, unhealthy child, suffering from deficient circulation. The entire palate was cleft, except the anterior part of the alveolar process. There had been a harelip, which had been closed in early life. Palatine arch very high; soft parts very scanty; articulation very imperfect.

Sept. 21st.-Under chloroform the soft palate was closed with four horsehair sutures. The palato-pharyngei were divided, and oblique cuts were made above to lessen the tension. At the end of a fortnight only the lowest part of the palate had united.

May 12th, 1869.-The child being in stronger health, under chloroform I closed the whole cleft in both the hard and soft palates. There was more tension than one would have wished; but this was owing to the scanty supply of soft parts. The wound was united by several silver sutures.

Four days after the operation one suture was removed on the fifth day three more were removed; and at the end of a week the rest were taken out.

July 6th. - The friends report that there is one very small hole in the hard palate, and one as large as a pea just where the hard and soft palate join. Should these holes not close by the contraction of cicatrisation, a small operation will be sufficient to obliterate them.

Cleft of soft palate in an adult.-W. C- aged twentyone, a healthy young man, was admitted into St. Bartholomew's Hospital on the 10th of April, 1869. 'The entire soft palate was cleft. The posterior margin of the hard palate was also notched; but here the deficiency was bridged over by soft parts. His power of articulation was unusually deficient for one in whom the soft palate only was affected. Under chloroform the palate was united, two horsehair sutures being used for the uvula, and four silver sutures for the palate. At the end of a week all the stitches were removed, and the palate was found completely united. In this case the palato-glossi and palato-pharyngei were greatly hypertrophied. They were, therefore, freely divided at the operation. The levator palati museles were not cut.

Cleft of the soft palate in an adult from syphilitic ulceration. -E. T_-, a girl, aged twenty, was admitted into St. Bartholomew's in May, 1869, suffering from a short, broad, median cleft of the soft palate; it measured about an inch in length, and resulted from ulceration occurring two years ago. Food and fluids were very apt to return through the nostrils, and the voice was nasal in tone.

May 14th. - The edges were pared and brought togethex by three horsehair sutures, and the palato glossi and pharyngei were divided. At the end of a week the patient was discharged cured.

Cleft of soft and hard palate in an adult.-A. H- - a woman, aged twenty-four, was admitted into St. Bartholomew's in October, 1867. She had complete cleft of the soft palate, and about half the hard; the cleft was unusually wide, and the soit parts scanty. Her articulation was very indistinct, and her voice most disagreeably resonant.

Oct. 30th.-I operated upon the soft palate under chloroform, closing it with four sutures of fishing gut, dividing the palato-pharyngei and the levatores palati muscles, and making oblique cuts above to relieve the tension. On the ninth day one suture was removed, and on the eighteenth day the others were taken out. The soft palate had united, but there remained a gap between the hard and soft palate measuring an inch and an eighth in length. Three months later this had contracted to less than five-eighths of an inch, when Mr. Coleman was good enough to make for her a vulcanite obturator.

To these instances of the use of chloroform in the cure of cleft palate I could adduce several more, derived from my personal experience. But these are sufficient to establish the fact that, with proper precautions, chloroform is as suitable to the performance of operations on the palate as it is to any other painful and protracted surgical proceeding. In confirmation of this statement I may refer to the experience of Mr. Paget, Mr. Gay, Mr. Adams, Mr. Holden, Mr. Savory, Mr. Callender, Mr. Langton, Mr. Annandale, and, I dare say, to many others who have employed the plan I recommend.

The monthly return of the births, deaths, and marriages, registered in the eight principal towns of Scotland, shows that during July the deaths were 253 above the average number, after allowing for increase of population, recorded for the same month during the last ten years ; 47 per cent. were those of children under five years of age. Diarrhoea, fever, and measles, were especially prevalent in Glasgow. Whooping-cough and scarlatina appear also to have been very rife, the former in Paisley particularly. 